\title{
Experimental study of extracorporeal lung resection in dogs: Ex situ sleeve resection and autotransplantation of the pulmonary lobe after extended pneumonectomy for central lung cancer
}

Isao Matsumoto, MD

Makoto Oda, MD

Yoshio Tsunezuka, MD

Masaya Tamura, MD

Kazuyuki Kawakami, MD

Go Watanabe, MD

From the Department of General and Cardio-thoracic Surgery, Kanazawa University, Kanazawa, Japan.

Supported by the Japanese Ministry of Education (No.08671519).

Received for publication July 25, 2003; revisions received Oct 14, 2003; accepted for publication Dec 2, 2003.

Address for reprints: Isao Matsumoto, MD, Department of General and Cardio-thoracic Surgery, Kanazawa University, Takara-machi 13-1, Kanazawa 920-8641, Japan (Email: mat@p2223.nsk.ne.jp).

J Thorac Cardiovasc Surg 2004;127:1343-9

$0022-5223 / \$ 30.00$

Copyright $\odot 2004$ by The American Association for Thoracic Surgery

doi:10.1016/j.jtcvs.2003.12.017
Objective: Extracorporeal lung resection as an alternative to pneumonectomy for central lung cancer is a procedure in which the unilateral lung is extirpated, removing the pulmonary lobe with the cancers and replanting the residual pulmonary lobe. The aim of this study was to investigate whether extracorporeal lung resection for lung cancer can be performed safely.

Methods: Nineteen dogs were divided into the control and extracorporeal lung resection groups. The former $(\mathrm{n}=5)$ underwent lung autotransplantion, and the latter was subdivided into ND1 $(n=7)$ and ND2 $(n=7)$ groups on the basis of the manner of lymph node dissection. By comparing the 3 groups, the adverse effects of lymph node dissection were examined.

Results: All dogs in the control group had no complications. Four dogs in the ND1 group survived for 90 to 630 days after the operation. In the ND2 group 5 dogs succumbed within 30 days after the operation, although the other 2 dogs survived for 391 and 573 days, respectively. Bronchopulmonary fistulas were seen in 1 of the ND1 dogs and 3 of the ND2 dogs. Two of the latter were free of thrombus formation in the pulmonary arteries and veins of the autografts. In the ND2 group, compared with the control and ND1 group, the tissue blood flow at the bronchial anastomotic site indicated reduction between the $3 \mathrm{rd}$ and 14 th postoperative days.

Conclusion: The extensive lymph node dissection had severe adverse effects on bronchial anastomotic healing in extracorporeal lung resection. Therefore extracorporeal lung resection can be applied to only a very limited number of patients with N0 or N1 disease.

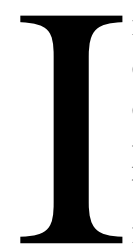

n many developed countries lung cancer has been the leading cause of cancer death. For non-small cell lung cancer, surgical removal of cancer cells is considered the only possible radical treatment. Treatment with pneumonectomy is inappropriate for some patients with dysfunctional lungs because this often leads to respiratory failure after the operation. The functional and technical problems often force them to seek other therapeutic modalities. As an alternative to pneumonectomy, some patients can adapt to extracorporeal lung resection (ECLR), ${ }^{1}$ in which the unilateral lung is extirpated, removing the pulmonary lobe with the cancers and replanting the residual pulmonary lobe into the body. However, only a few examples of ECLR have been reported, ${ }^{1-3}$ 
and this procedure has yet to undergo the extensive investigation required before it can be accepted as an established treatment.

There are several advantages to ECLR. First, because it involves autotransplantation, the immunologic system does not reject the replanted tissue. Additionally, ECLR can be performed in a favorable visual field without excessive bleeding because the pulmonary lobe with the cancers can be removed from the body. With this method, it is easier to recognize with some consistency the area infiltrated by a cancerous growth and the condition of the lesions. However, there are also problems associated with ECLR, including the development of bronchopulmonary fistulas (BPFs). ${ }^{1-3}$ Unlike conventional lung transplantation, ECLR for lung cancer requires lymph node dissection. There is a great need for research into subsequent adverse effects, but very little has been reported on this subject.

The aim of this study was to investigate whether ECLR for lung cancer can be performed safely. In this series the adverse effects of lymph node dissection in ECLR were evaluated.

\section{Materials and Methods}

\section{Animals}

Humane care was provided for all dogs in accordance with the "Guide for the Care and Use of Laboratory Animals" published by the National Institutes of Health (National Institutes of Health publication no. 86-23, revised 1985) and the guidelines of the Japanese Association of Experimental Animals.

\section{Surgical Procedure}

Nineteen adult mongrel dogs, each weighing 8.5 to $16 \mathrm{~kg}$ (average, $12.9 \mathrm{~kg}$ ), were premedicated and anesthetized with $0.02 \mathrm{mg} / \mathrm{kg}$ atropine sulfate and $10 \mathrm{mg} / \mathrm{kg}$ ketamine hydrochloride. After placing an instiller in the cubital vein, anesthesia was maintained with $30 \mathrm{mg} / \mathrm{kg}$ pentobarbital administered intravenously when necessary. After endotracheal intubation, the dogs were ventilated with a volume-cycle ventilator to keep the respiratory volume at 25 $\mathrm{mL} / \mathrm{kg}$ (with unilateral lung ventilation, $15 \mathrm{~mL} / \mathrm{kg}$ ). During the operation, the fraction of inspiratory oxygen was maintained at $50 \%$. Before and after the operation, antibiotics were administered.

\section{Experimental Protocol}

The dogs were divided into control and ECLR groups. The former ( $\mathrm{n}=5$ ) underwent lung autotransplantion, and the latter were subdivided into ND1 $(n=7)$ and ND2 $(n=7)$ groups on the basis of the manner of lymph node dissection. By comparing the 3 groups, the adverse effects of lymph node dissection were examined. We selected the left lung for autotransplantation and ECLR because in dogs the right lung consists of 4 lobes and the left lung consists of 3 lobes. As shown in Figure 1, the configuration of the left lung provides easier handling for experiments.

Control group. A left posterolateral skin incision was made, and a thoracotomy was performed. After intravenous injection of $100 \mathrm{U} / \mathrm{kg}$ heparin sodium, the left lung was extirpated (Figure 1, A and $B$ ) and then replanted. The left main bronchus was resected at
2 bronchial rings proximal to the site where the cranial and caudal bronchi bifurcate. During the extirpation procedure, the left lung was expanded with a pressure of $15 \mathrm{~cm} \mathrm{H}_{2} \mathrm{O}$. After Euro-Collins solution with $2000 \mathrm{U}$ of heparin sodium was perfused through the pulmonary artery, the lung was preserved for 1 hour. Then the lung underwent end-to-end anastomosis to the pulmonary vein, bronchus, and pulmonary artery. Lymph nodes were not dissected.

ECLR group. The left lung was extirpated by using the same method as that used in the control group. After Euro-Collins solution with $2000 \mathrm{U}$ of heparin sodium was perfused through the pulmonary artery, the lung was divided into the cranial and caudal lobes (Figure 1,C). After 1 hour of extracorporeal preservation, the caudal lobe was implanted (Figure 1,D).

Lymph node dissection in the ECLR group. Lymph node dissection was performed according to Anatomy of the Dog, ${ }^{4}$ which shows that there are anatomic similarities between human subjects and dogs in the bronchial blood and lymph supply. The ND1 group underwent a hilar lymph node dissection. The hilar lymph nodes (left bronchial lymph nodes) were removed together with the extirpated lung and dissected when the lungs were separated. In the ND2 group systematic hilar and mediastinal lymph node dissection (cranial mediastinal lymph nodes and left and middle tracheobronchial lymph nodes, including the surrounding fatty tissues) was performed. Resection of the mediastinal lymph nodes was confined to the cranial mediastinal lymph nodes as the cephalad limit, the pulmonary ligament as the caudal limit, the thymus as the anterior limit, and the anterior end of the line of the descending aorta as the posterior limit.

Assessment of autograft. Chest radiographs and bronchoscopic findings were obtained on the 3rd, 7th, 14th, 21st, and 60th postoperative days (PODs).

To objectively assess the degree of pulmonary autograft injury on chest radiographs, we used a semiquantitative scale according to the method of Aziz and colleagues, ${ }^{5}$ with scoring as follows: 0, no abnormal findings; 1 , perihilar infiltration; 2 , infiltration localized to a limited lung field; 3, diffuse moderate interstitial and alveolar infiltration; and 4, diffuse severe interstitial and alveolar infiltration. The 3 groups were compared by using the means of scores.

To evaluate the anastomotic site healing, we used the classification of Couraud and associates, ${ }^{6}$ with bronchoscopic findings as follows: grade 1, complete circumferential primary mucosal healing; grade $2 \mathrm{~A}$, complete circumferential healing but partial mucosal healing; grade $2 \mathrm{~B}$, no mucosal healing; grade $3 \mathrm{~A}$, focal necrosis; and grade $3 \mathrm{~B}$, extensive necrosis.

We used the airway anastomotic index (AAI) to assess the blood flow at the bronchial anastomotic site. Blood flow of the bronchial mucosa was measured by using a laser tissue blood flowmeter (in milliliters per minute per 100 grams; Advans, Tokyo, Japan) through a bronchoscope. This technique can be useful for evaluating the effect of bronchial artery revascularization on mucosal blood flow and can be a prognostically useful tool in follow-up of bronchial anastomotic healing. ${ }^{7}$ The measuring sites were the membranous area very close to the bronchial anastomotic site of the autograft side and the area of the left main bronchus 2 bronchial rings distal to the tracheal bifurcation. We defined the former as an anastomotic site and the latter as a control site. Measurement was conducted 3 times at each site, and the mean 

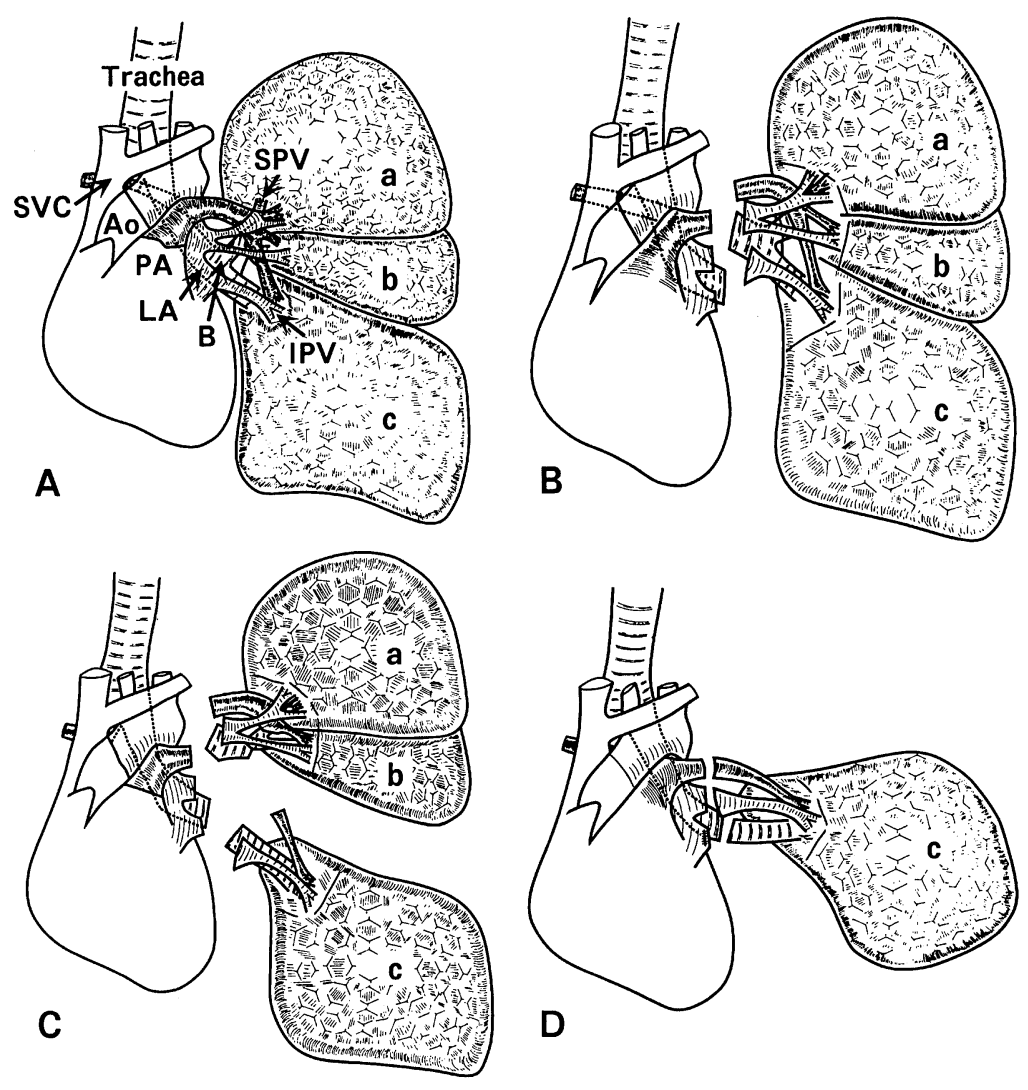

Figure 1. The scheme of the procedure for ECLR (A-D). The left lung was divided into the cranial and caudal lobes after pneumonectomy. After 1 hour of extracorporeal preservation, the caudal lobe was implanted. SVC, Superior vena cava; $A$ o, aorta; $P A$, pulmonary artery; $L A$, left atrium; $B$, bronchus; $S P V$, superior pulmonary vein; $I P V$, inferior pulmonary vein; $a$, cranial part of cranial lobe of left lung; $b$, caudal part of cranial lobe of left lung; $c$, caudal lobe of left lung.

was obtained. Thereafter, we calculated the ratio of blood flow at the anastomotic site to that at the control site in all surviving dogs over various time periods. This ratio was expressed as the AAI. We used the relative values because blood flow depends on the hemodynamic status.

Measurement of $\mathrm{PaO}_{2}$ and pulmonary arterial pressure and pulmonary angiographic findings. $\mathrm{PaO}_{2}$ and pulmonary arterial pressure (PAP) were measured before killing the dogs. Pulmonary angiographs of the lungs were prepared after they were excised from the killed dogs. On the basis of our findings, we decided whether the stenosis was located at the anastomotic site of the pulmonary artery and vein.

Histopathologic analysis. In each group the autografts, including the anastomotic sites of the pulmonary artery, vein, and bronchus, were extirpated at the time of death. After the lung had been fixed with a $10 \%$ formalin solution, paraffin sections of the anastomotic sites on the pulmonary artery, vein, bronchus, and peripheral pulmonary tissue were prepared and subjected to hematoxylineosin staining and Elastica van Gieson staining.

\section{Statistical Analysis}

The results of the experiments were expressed as the average score $\pm \mathrm{SD}$. For comparative examination, ischemic time, chest radio- graphic score, AAI, $\mathrm{PaO}_{2}$, and PAP were analyzed by using the Mann-Whitney $U$ test.

\section{Results}

\section{Mean Ischemic Time}

The mean ischemic times between extirpation and implantation of the lung were $145 \pm 19,135 \pm 31$, and $141 \pm 26$ minutes in the control, ND1, and ND2 groups, respectively. There was no significant difference among these groups.

\section{Results of the Operation}

Postoperative complications, length of survival, and cause of death (diagnosed after autopsy) for each group are detailed in Table 1. In the control group 4 dogs survived for 57 to 168 days without complications until they were killed. One dog succumbed to asthenia without an obvious cause. In the ND1 group 3 dogs died: 1 each from pneumonia, BPF, and asthenia. The remaining 4 dogs survived for 90 to 630 days. The pulmonary artery and vein of the autograft in $\operatorname{dog} 6$, which succumbed to BPF, were obstructed by thrombi. In the ND2 group 1 dog died from pneumonia, 3 
TABLE 1. Results of the operation

\begin{tabular}{lcrcccl}
\hline Group & $\begin{array}{c}\text { Dog } \\
\text { no. }\end{array}$ & $\begin{array}{c}\text { Survival } \\
\text { (d) }\end{array}$ & BPF & $\begin{array}{c}\text { Thrombi } \\
\text { in PV }\end{array}$ & $\begin{array}{c}\text { Bronchial } \\
\text { stenosis }\end{array}$ & $\begin{array}{c}\text { Cause of death } \\
\text { (autopsy) }\end{array}$ \\
\hline Control & 1 & 66 & - & - & - & Killed \\
& 2 & 18 & - & - & - & Asthenia \\
& 3 & 168 & - & - & - & Killed \\
& 4 & 57 & - & - & - & Killed \\
ND1 & 5 & 79 & - & - & - & Killed \\
& 1 & 630 & - & - & - & Killed \\
& 2 & 608 & - & - & - & Killed \\
& 3 & 12 & - & - & - & Pneumonia \\
& 4 & 32 & - & - & - & Asthenia \\
& 5 & 90 & - & - & - & Killed \\
& 6 & 31 & + & + & - & BPF, pyothorax \\
ND2 & 7 & 478 & - & - & - & Killed \\
& 1 & 1 & - & - & - & Bleeding \\
& 2 & 10 & + & - & - & BPF \\
& 3 & 7 & - & - & - & Pneumonia \\
& 4 & 16 & + & + & - & BPF \\
& 5 & 573 & - & - & - & Killed \\
& 6 & 26 & + & - & - & BPF \\
& 7 & 391 & - & - & + & Killed \\
\hline
\end{tabular}

$\overline{B P F}$, Bronchopulmonary fistula; $P V$, pulmonary vein; Control, lung autotransplantation without lymph node dissection; ND1, extracorporeal lung resection with hilar lymph node dissection alone; $N D 2$, extracorporeal lung resection with systematic hilar and mediastinal lymph node dissection.

died from BPF, and 1 died from bleeding. Of the 2 dogs that survived for a long period, 1 showed bronchial stenosis caused by granulation tissue at the anastomotic site. At the autopsy on the 391st day, the anastomotic site was almost occluded. The other dog survived for 573 days after the operation. Analysis of the 3 dogs that succumbed to BPF showed that the pulmonary artery and vein of the autograft in $\operatorname{dog} 4$ were obstructed by thrombi. The other 2 dogs were free of thrombus formation.

\section{Chest Radiograph Score}

The mean chest radiograph scores of surviving dogs in each group over various time periods are shown in Table 2. On the third and seventh PODs, pulmonary injury was significantly more severe in the ND2 group than in the control or ND1 groups (third POD, $P=.05$ vs the control and ND1 groups; seventh POD, $P=.01$ vs the control group and $P=$ .02 vs the ND1 group). There were no significant differences between the control and ND1 groups. One of the ND2 dogs that survived for a long period retained its respiratory functions until the 60th POD, but its lungs became atelectatic after 1 year.

\section{Grading of Anastomotic Site Healing}

In the control group Couraud grading of anastomotic site healing on the seventh POD was $2 \mathrm{~A}$ in all dogs. After the 14th POD, all dogs were grade 1. In the ND1 group on the seventh POD, 6 dogs were grade $2 \mathrm{~A}$, and 1 dog was grade
TABLE 2. Chest radiograph scores

\begin{tabular}{lccccc}
\hline & \multicolumn{5}{c}{ Postoperative time (d) } \\
\cline { 2 - 6 } Group & 3rd & 7th & 14th & 21st & 60th \\
\hline Control & $1.6 \pm 0.5^{*}$ & $1.2 \pm 0.4 \dagger$ & $0.2 \pm 0.4$ & $0.2 \pm 0.4$ & 0.0 \\
ND1 & $1.7 \pm 0.5$ & $1.3 \pm 1.0$ & $0.7 \pm 1.2$ & $0.7 \pm 1.6$ & 0.0 \\
ND2 & $2.3 \pm 0.5$ & $2.6 \pm 0.5$ & $1.8 \pm 0.8$ & $0.7 \pm 1.2$ & 0.0 \\
\hline
\end{tabular}

Values are presented as means \pm SD. See Table 1 for definitions of groups.

${ }^{*} P=.05$, compared with the control group and the ND1 group.

$\dagger P=.01$ compared with the control group; $P=.02$ compared with the ND1 group.

3B. After the 14th POD, all surviving dogs were grade 1. In the ND2 group on the seventh POD, 2 dogs were grade $2 \mathrm{~B}$, $1 \mathrm{dog}$ was grade $3 \mathrm{~A}$, and $2 \mathrm{dogs}$ were grade $3 \mathrm{~B}$. Reepithelization was barely detectable on the third to seventh PODs. On the 14th POD, 2 dogs were grade 2A, 1 dog was grade $3 \mathrm{~A}$, and $1 \mathrm{dog}$ was grade $3 \mathrm{~B}$. Two dogs that survived for a long period were grade 1 after the 21st POD. However, bronchial stenosis gradually developed in $\operatorname{dog} 7$, and the lesion was almost occluded by granulation tissue after 1 year. Compared with the control and ND1 groups, re-epithelization in the ND2 group was delayed.

\section{Airway Anastomotic Index}

Average AAI values of each group over various time periods are shown in Figure 2. For the control group, the AAI was $69.5 \% \pm 10.3 \%$ on the third POD but recovered to $82.7 \% \pm 9.3 \%$ on the seventh POD. In the ND1 group the AAI was $62.6 \% \pm 9.6 \%$ on the third POD and recovered to $80.5 \% \pm 4.2 \%$ on the seventh POD. In the ND2 group the AAI was markedly reduced on the third POD $(31.6 \% \pm$ 9.5\%), and remained low on the seventh POD (44.6\% \pm $14.6 \%$ ). This condition gradually improved to $71.0 \% \pm$ $20.9 \%$ and $89.2 \% \pm 16.1 \%$ on the 14 th and 21 st PODs, respectively. On the 60th POD, the 3 groups had equal AAI values. Compared with the control and ND1 groups, the AAI of the ND2 group was significantly reduced on the third $(P=.0001$ vs the control and ND1 groups) and seventh ( $P=.001$ vs the control and ND1 groups) PODs. There were no significant differences between the control and ND1 groups.

\section{Measurement of $\mathrm{PaO}_{2}$ and PAP and Angiographic Findings}

The mean preoperative $\mathrm{PaO}_{2}$ values of the control, ND1, and $\mathrm{ND} 2$ groups were $93.7 \pm 5.8 \mathrm{~mm} \mathrm{Hg}, 92.9 \pm 5.2 \mathrm{~mm} \mathrm{Hg}$, and $92.7 \pm 4.7 \mathrm{~mm} \mathrm{Hg}$, respectively. The mean $\mathrm{PaO}_{2}$ values of the dogs that remained alive for a long period in each group were $93.3 \pm 5.4 \mathrm{~mm} \mathrm{Hg}, 91.0 \pm 4.4 \mathrm{~mm} \mathrm{Hg}$, and $90.5 \pm 4.7 \mathrm{~mm} \mathrm{Hg}$, respectively. There were no significant differences between the groups before the operation, and 


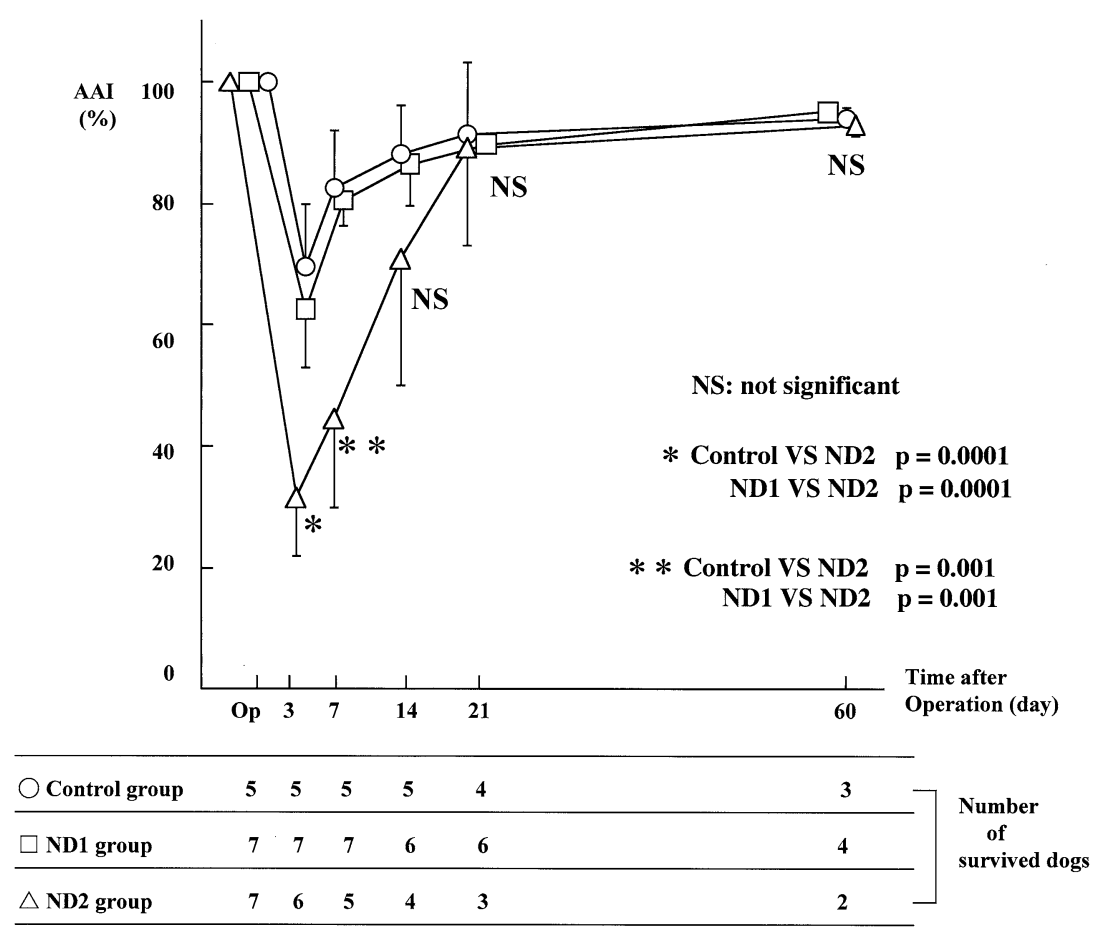

Figure 2. Changes in AAl: circles, control group; squares, ND1 group; triangles, ND2 group. We calculated the ratio of blood flow at the anastomotic site to that at the control site (the area of the left main bronchus 2 bronchial rings distal to the tracheal bifurcation) in all surviving dogs over various time periods. This ratio was expressed as the AAI.

there were no significant differences between before and after the operation within either group.

The right mean PAPs of the control, ND1, and ND2 groups were $13.5 \pm 2.2 \mathrm{~mm} \mathrm{Hg}, 12.8 \pm 1.8 \mathrm{~mm} \mathrm{Hg}$, and $12.6 \pm 1.2 \mathrm{~mm} \mathrm{Hg}$, respectively. The left mean PAPs were $15.1 \pm 3.9 \mathrm{~mm} \mathrm{Hg}, 15.2 \pm 2.4 \mathrm{~mm} \mathrm{Hg}$, and $16.5 \pm 1.7 \mathrm{~mm}$ $\mathrm{Hg}$, respectively. There was a tendency for the left mean PAP to be slightly higher than the right mean PAP in all groups.

All dogs that survived for a long time showed fine arterial and venous conditions. There was no stenosis or twisting of the pulmonary artery or vein.

\section{Histologic Findings in the Dogs That Survived for a Long Period}

At the bronchial anastomotic sites, the bronchial epithelium at the anastomotic site was completely re-epithelialized in all groups.

At the pulmonary arterial and venous anastomotic sites, in all groups regeneration of the endothelial cells was complete at the anastomotic sites. Dense fibrous cicatricial tissue had formed around these sites.

At the peripheral pulmonary tissue, the findings were identical in all groups. Some emphysematous changes were noted, but the alveolar structure was preserved. There were no cases of thickening of the interalveolar septa or inflammatory cellular infiltration. The visceral pleura were generally thickened with fibrous tissue.

\section{Discussion}

ECLR, as an alternative to pneumonectomy for patients with central lung cancer and a dysfunctional lung, was first reported by Vogt-Moykopf in 1992. ${ }^{1}$ This radical therapeutic approach to lung cancer helps preserve pulmonary function. ECLR can be used in cases in which it is difficult to remove the tumor even by means of pulmonary lobectomy or sleeve lobectomy. Specifically, the procedure might be applicable to cases in which the tumor infiltrates the pulmonary artery and vein or in cases in which it is difficult to separate the tumor from normal tissues because the tumor is complicated by a hilar inflammatory disease, such as pulmonary tuberculosis.

In many reported series ${ }^{8-13}$ complications and mortalities of sleeve lobectomy are less severe than those of pneumonectomy. Long-term survival is certainly comparable and in some reported series ${ }^{10,11}$ is higher for sleeve lobectomy. It has been reported that patients who have had pneumonectomy have a higher death rate from non-cancer-related causes. In this respect, by preserving pulmonary function, 
ECLR, compared with pneumonectomy, might reduce the incidence of respiratory failure; ECLR is an advanced sleeve lobectomy technique.

With ECLR, the immunologic system does not reject the graft, but there are often complications at the anastomotic sites, and this increases operative mortality. ${ }^{1-3}$ There is a great need to eliminate these problems. In partial lung transplantation, major causes of complications at the bronchial anastomotic site are stenosis and obstruction caused by thrombus formation at the anastomosed vein. ${ }^{14,15}$ Stenosis in the pulmonary vein results in pulmonary edema and obstruction of the pulmonary artery. During the early period after an operation, the peripheral bronchus of the bronchial anastomotic site is nurtured by backflow from the pulmonary artery. ${ }^{16}$ Thus stenosis at the pulmonary vein often leads to BPF, as observed in the ND1 and ND2 groups. For these reasons, special care should be taken to prevent stenosis at the anastomotic site of the pulmonary vein. It might be necessary to consider systemic anticoagulation treatment after the operation, ${ }^{17}$ although anticoagulant was not administered after the operation in the present study because it is not usually necessary in autotransplantation models.

ECLR to treat lung cancer must be accompanied by systematic lymph node dissection, including excision of the mediastinal lymph nodes. To our knowledge, there have been no reports that describe how lymph node dissection influences the bronchial anastomotic site healing in lung transplantation. From the 3rd to the 14th POD, the tissue blood flow at the bronchial anastomotic site was lower in the ND2 group than in the control or ND1 groups. During this period, in the ND2 group the bronchial anastomotic site healing was delayed, and the pulmonary injury was severe. BPF without obstruction in the pulmonary arteries and veins might be due to the extensive lymph node dissection, leading to decreased blood flow at the bronchial anastomotic site and diminished lymphatic flow. At this site, circulation might become very poor because the microcirculation in the pulmonary peripheral vessels is further curtailed by posttransplantation reperfusion injury. ${ }^{18}$ The present canine model was not a lung cancer model, and the dogs were not exposed to the adverse effects of smoking. Although dogs with healthy lungs were selected, the failure rate was high in the ND2 group. For patients with low respiratory functions, complications can lead to death. Therefore ECLR cannot be applied to a wide range of patients, especially those with $\mathrm{N} 2$ disease.

On the other hand, the prognosis of pathologic N2 nonsmall cell lung cancer is generally poor. Also, for patients with N0 or N1 status, survival after sleeve lobectomy is equal to or better than that of pneumonectomy for a similarstage tumor. ${ }^{10}$ Therefore ECLR should also be used to treat NO and N1 non-small cell lung cancers, considering the factors of lymph node dissection and prognosis. Actual clinical application of ECLR might be limited to localized central lung cancer, which cannot be treated with pulmonary lobectomy or sleeve lobectomy, after excluding N2 disease by means of computed tomography, positron emission tomography, or mediastinoscopy. ${ }^{19}$ However, ECLR might be indicated for some patients, although probably a very limited number. Thus we conclude that the technique can be a useful procedure in certain cases.

The limitation of this study is that we used dogs that underwent autotransplantation of an entire lung as a control group. We selected this simple control to evaluate bronchial anastomotic healing and blood flow at the bronchial anastomotic site in autotransplantation, excluding the technical and hemodynamic factors in autotransplantation of a pulmonary lobe. It would be much better for the control group to comprise dogs that underwent autotransplantation of a lobe. However, although the ND1 group did not have significantly different bronchial healing than the control group, there were obvious adverse effects in the ND2 group. Additionally, the present results raise the following question: Can using viable tissues to cover the bronchial anastomotic site improve the blood flow at the bronchial anastomotic site? Experiments should be designed to investigate whether such innovative methods can improve the bronchial anastomotic healing.

\section{References}

1. Vogt-Moykopf I, Krysa S, Schirren J. Aufgaben und Zukunftsaspekte der Thoraxchirurgie. In: Vogt-Moykopf I, editor. Thoraxchirurgie: Stand und Ausblic. Darmstadt, Germany: Steinkopff Verlag; 1993, p. 142-3.

2. Hendrix H, Schmitt J, Aebert H. Ex situ sleeve resection and autotransplantation of the left lower pulmonary lobe after extended pneumonectomy. Chirurg. 2000;71:820-3.

3. Zhang G, Liu J, Jiang G, Shen C, Li M. Lung autotransplantation technique for treating central lung cancer of upper lobe. Zhonghua Wai Ke Za Zhi. 2000;38:245-9.

4. Budras K, McCarthy P. Thoracic cavity. In: Budrus KD, editor. Anatomy of the dog: an illustrated text. 4th ed. Hannover, Germany: Schlutersche GmbH \& Co. KG, Verlag und Druckerei; 2002. p. 38-49.

5. Aziz TM, Pillay TM, Corris PA, Forty J, Hilton CJ, Hasan A, et al. Perfadex for clinical lung procurement: is it an advance? Ann Thorac Surg. 2003;75:990-5.

6. Couraud L, Nashef SA, Nicolini P, Jougon J. Classification of airway anastomotic healing. Eur J Cardiothorac Surg. 1992;6:496-7.

7. Hyytinen TA, Halme M. Laser Doppler flowmetry detects early risk of tracheal anastomotic complications after lung transplantation. Scand Cardiovasc J. 2000;34:345-9.

8. Tedder M, Anstadt MP, Tedder SD, Lowe JE. Current morbidity, mortality, and survival after bronchoplastic procedures for malignancy. Ann Thorac Surg. 1992;54:387-91.

9. Ginsberg RJ, Hill LD, Eagan RT, Thomas P, Mountain CF, Deslauriers $\mathrm{J}$, et al. Modern thirty-day operative mortality for surgical resections in lung cancer. J Thorac Cardiovasc Surg. 1983;86: 654-8.

10. Tronc F, Grégoire J, Rouleau J, Deslauriers J. Long-term results of sleeve lobectomy for lung cancer. Eur J Cardiothorac Surg. 2000;17:550-6.

11. Jensik RJ, Faber LP, Kittle CF. Sleeve lobectomy for bronchogenic carcinoma: the Rush-Presbyterian-St. Luke's Medical Center experience. Int Surg. 1986;71:207-10.

12. Martin-Ucar AE, Chaudhuri N, Edwards JG, Waller DA. Can pneu- 
monectomy for non-small cell lung cancer be avoided? An audit of parenchymal sparing lung surgery. Eur J Cardiothorac Surg. 2002;21: 601-5.

13. Terzi A, Lonardoni A, Falezza G, Furlan G, Scanagatta P, Pasini F, et al. Sleeve lobectomy for non-small cell lung cancer and carcinoids: results in 160 cases. Eur J Cardiothorac Surg. 2002;21:888-93.

14. Crombleholme TM, Adzick NS, Longaker MT, Bradley S, Duncan BW, Verrier ED, et al. Reduced-size lung transplantation in neonatal swine: technique and short-term physiological response. Ann Thorac Surg. 1990;49:55-60.

15. Kern JA, Tribble CG, Chan BBK, Flanagan TL, Kron IL. Reducedsize porcine lung transplantation: long-term studies of pulmonary vascular resistance. Ann Thorac Surg. 1992;53:583-9.
16. Ishihara T, Nemoto E, Kikuchi K, Kato K, Kobayashi K. Does pleural bronchial wrapping improve wound healing in right sleeve lobectomy? J Thorac Cardiovasc Surg. 1985;89:665-72.

17. Sarsam MA, Yonan NA, Beton D, McMaster D, Deiraniya AK. Early pulmonary vein thrombosis after single lung transplantation. J Heart Lung Transplant. 1993;12:17-9.

18. Miller DL, Roberts AM. Pulmonary artery occlusion and reperfusion causes microvascular constriction in the rabbit lung. Ann Thorac Surg. 1999;67:323-8.

19. Kernstine KH, Mclaughlin KA, Menda Y, Rossi NP, Kahn DJ, Bushnell DL, et al. Can FDG-PET reduce the need for mediastinoscopy in potentially resectable nonsmall cell lung cancer? Ann Thorac Surg. 2002; 73:394-401.

\section{Bound volumes available to subscribers}

Bound volumes of The Journal of Thoracic and Cardiovascular Surgery are available to subscribers (only) for the 2004 issues from the Publisher, at a cost of $\$ 134.00$ for domestic, $\$ 165.85$ for Canadian, and $\$ 155.00$ for international subscribers for Vol 127 (January-June) and Vol 128 (July-December). Shipping charges are included. Each bound volume contains a subject and author index and all advertising is removed. The binding is durable buckram with the Journal name, volume number, and year stamped in gold on the spine. Payment must accompany all orders. Contact Elsevier Inc., Subscription Customer Service, 6277 Sea Harbor Dr, Orlando, FL 32887, USA; phone 800-654-2452 or 407-345-4000.

Subscriptions must be in force to qualify. Bound volumes are not available in place of a regular Journal subscription. 